\title{
DIFFERENTIATION OF HIPPELATES PUSIO, H. BISHOPPI AND H. PALLIPES (DIPTERA: CHLOROPIDAE) BY ELECTROPHORESIS ${ }^{1}$
}

\author{
By Frederic M. Stiner ${ }^{2,3}$, F. M. Johnson ${ }^{2,4}$ and Richard C. Axtell ${ }^{2,5}$
}

Abstract: Hippelates pusio Loew, H. bishoppi Sabrosky, and $H$. pallipes (Loew) (Diptera: Chloropidae) from North Carolina were examined by starch gel electrophoresis for 5 enzymes: esterase, $\alpha$-glycerophosphate dehydrogenase, "leucine" aminopeptidase, NAD dependent malic dehydrogenase, and aldehyde oxidase. Both similarities and differences in electrophoretic patterns among the species were noted. The esterase and aminopeptidase patterns from larval extracts apparently were distinguishing characteristics of all 3 species. The other enzymes were less species-specific.

Hippelates eye gnats (Diptera: Chloropidae) have been implicated as vectors of pink-eye (Dow \& Hines 1957) and bovine mastitis (Sanders 1940) in the southeastern U. S., and their presence is an annoyance to man and animals (Dow et al. 1951). Some members of the $H$. pusio Loew species group, as delineated by Sabrosky (1941), are most commonly involved. Three species of this group are abundant in North Carolina (Axtell \& Edwards 1970): H. pusio, H. bishoppi Sabrosky, and H. pallipes (Loew). Adult forms of all 3 are similar morphologically, but they can be distinguished by using Sabrosky's (1941) key. Terminology, however, has often caused confusion (Sabrosky 1951) and differentiating $H$. pusio from $H$. bishoppi is difficult. Identification of these 2 species depends upon leg color, dorsal setation between the median and dorsocentral bristles, and relative angle of the hind tibial spur (Sabrosky 1941). We have found that leg color varies from black to yellow in both $H$. pusio and H. bishoppi. Dorsal setation is more reliable: 3 rows between the median and dorsocentral bristles in $H$. pusio and 1 row in the same area in $H$. bishoppi. Using the hind tibial spur for identification requires considerable experience. No characters for separation of the immature stages of species of Hippelates are known.

In the present study, further characters to separate these species were sought by an isozyme analysis of the various life stages (Markert \& Moller 1959).

\footnotetext{
${ }^{1}$ Supported by PHS Training Grant ES00069 and PHS Research Grant No. GM 11546, and Contract No. AT-(40-1)3980 with the U. S. Atomic Energy Commission. Paper No. 3299 of the Journal Series of the North Carolina State University Agricultural Experiment Station, Raleigh.

${ }^{2}$ North Carolina State University, Raleigh, N. C. 27607, U.S.A.

${ }^{3}$ USPHS Predoctoral Trainee, Dept. of Entomology.

${ }^{4}$ Asst. Professor, Dept. of Genetics.

${ }^{5}$ Professor, Dept. of Entomology.
}

Isozyme analysis has recently been applied to the determination of biochemical differences among different species of Drosophila (Diptera: Drosophilidae) by Johnson et al. (1966, 1968, 1969).

\section{MATERIALS AND METHODS}

The flies used in these experiments were obtained from the laboratory colonies described by Karandinos \& Axtell (1967). These colonies originated from flies collected in 1963-1964 in North Carolina.

The major part of this investigation was conducted with adult male and female flies of various ages and third-instar larvae. Preliminary experiments produced better results with these stages than with eggs, early instar larvae, or pupae. Whole individuals were individually homogenized in cylindrical chambers in a cold Delrin ${ }^{\circledR}$ plastic block with a powerdriven $.317 \mathrm{~cm}(1 / 8$ in.) stainless steel rod. This was a modification of Johnson's (1966) procedure. Horizontal starch gel electrophoresis was used with the gels cooled by being in contact with a tray of ice. Two different buffer systems were used: (1) the discontinuous system of Poulik (1957) and (2) a continuous system containing $0.135 M$ Tris, 0.043 $M$ citrate, $\mathrm{pH} 7.0$ in the electrode vessels and 0.009 $M$ Tris, $0.0029 M$ citrate, $\mathrm{pH} 7.0$, in the gels. An initial current of 100 ma was used (Heath Kit model IP-17 power supply) and current flow was stopped after the front was approximately $8 \mathrm{~cm}$ from the origin. Dimensions of the gels were $19.7 \times 17.2 \times 1.0 \mathrm{~cm}$.

Following the migration, the gels were stained for 5 different enzyme activities: esterase (EST), "leucine" aminopeptidase (LAP), NAD-dependent malic dehydrogenase (MDH), aldehyde oxidase $(\mathrm{AOX})$, and $\alpha$-glycerophosphate dehydrogenase $(\alpha-$ GPDH), according to Johnson et al. (1969). The esterase stain contained 2 substrates, $\alpha$ - and $\beta$ napthyl acetate, and it allowed detection of some differences in esterase substrate specificity (Johnson et al. 1966). About 50 individuals were examined for each of the 5 enzyme activities after preliminary investigation revealed which life stage gave the clearest separation for each enzyme.

RESULTS AND DISCUSSION

Clarity of isozyme analysis depends to a large 

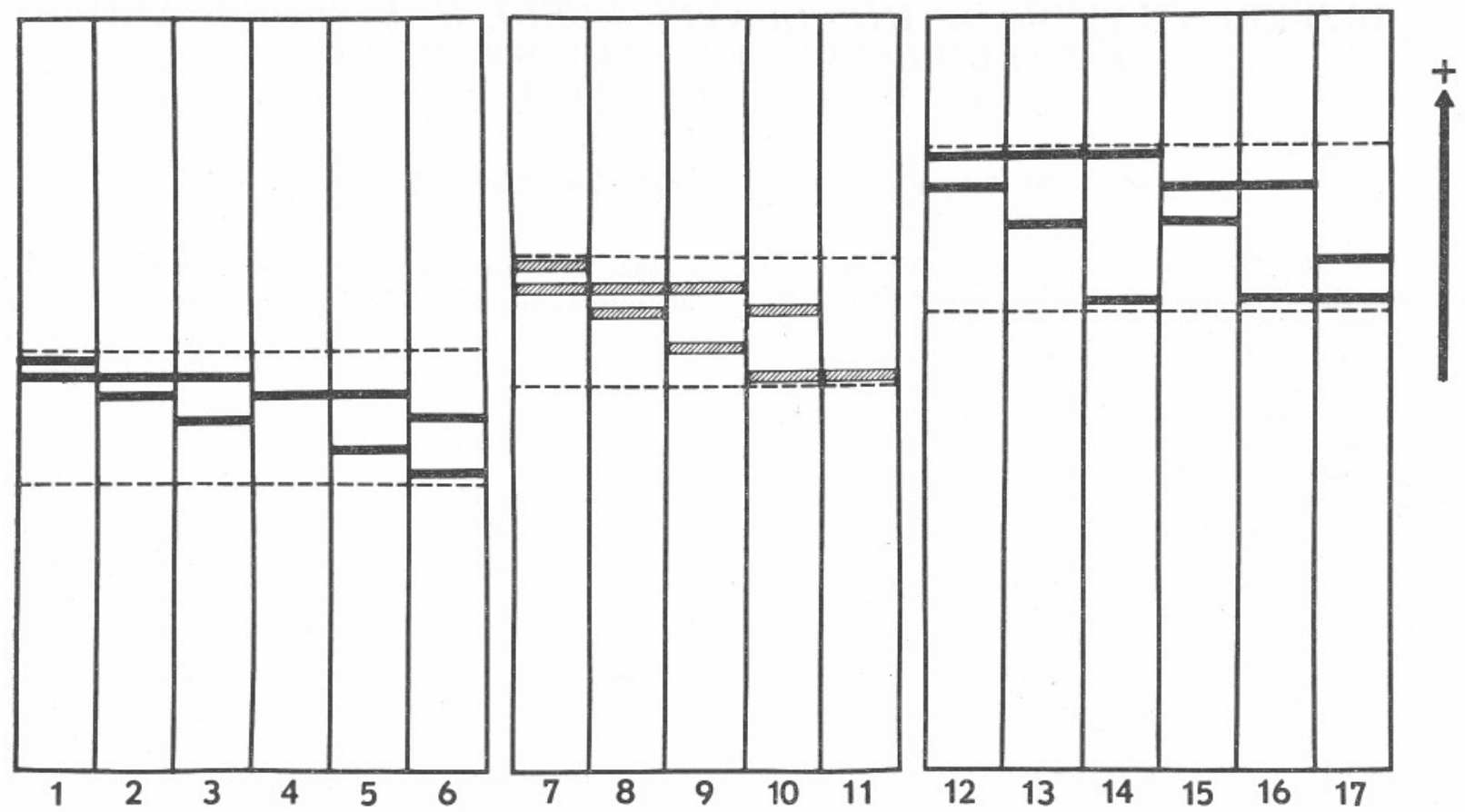

FIG. 1. Diagrammatic comparison of the esterase patterns of larvae for H. pusio (1-6), H. bishoppi (7-11), and $H$. pallipes (12-17). The esterase bands in each species vary in mobility and typical examples are illustrated. The horizontal dashed lines indicate the zones within which the bands occurred. Solid shading of bands indicates $\beta$-substrate specificity (red bands) and slanted-line bands indicate that both $\alpha$ - and $\beta$ - substrates were hydrolyzed (purple bands). Migration is in the direction indicated by the arrow in all figures.

degree on the buffer system as well as on the life stage used. For EST, larvae were the best staining stage, and the Poulik (1957) buffer gave the best resolution. The results obtained with pupae and adults could not be reproduced, and these stages were not used beyond preliminary examination. Typical variation within and among the zymograms of the 3 species is diagrammed in FIG. 1. Each of the 3 species showed mainly a single "zone" of EST activity. Each zone in the 3 species was a complex pattern of bands, the bands being either single or double.

$H$. pusio and $H$. pallipes can be distinguished by the relative mobilities of the esterase zones. The $H$. pallipes zone had a greater mobility than that of $H$. pusio, but it is possible that this difference is a reflection of the restricted genetic variation in the colonies. The bands making up the EST zone in $H$. bishoppi were intermediate and overlapping in mobility to those of $H$. pusio and $H$. pallipes. However, there was a difference in the substrate specificity of the $H$. bishoppi esterases which differentiated this species from the other 2. In $H$. bishoppi the esterase bands stained purple in color while in $H$. pusio and $H$. pallipes they stained red. Band color is indicative of substrate specificity.
When bands are red this indicates that the esterases have hydrolyzed mostly the B-napthol ester, when they are blue-black mostly $\alpha$-ester has been hydrolyzed, and when they are purple, both esters have been hydrolyzed (cf. Johnson et al. 1966). Other minor EST zones also appeared in the zymograms, but they were only faintly visible and were not investigated.

For the analysis of LAP, only larvae provided acceptable and consistently clear enzyme patterns. The Poulik (1957) buffer was found to produce the best differentiation of enzyme bands. The LAP banding patterns for the 3 species are diagrammed in FIG. 2. Each species had one densely staining LAP band plus a number of faint bands. There was little difference among the species in regard to the mobilities of the faint bands, but all 3 species showed a different mobility for their most dense LAP band. Thus the mobility of this band apparently allows one to distinguish the 3 species. No variation of the type found in EST was noted for any of the LAP bands.

The typical isozyme pattern for $\alpha-$ GPDH was a single band of activity (FIG. 3). Only adults could be satisfactorily stained for $\alpha-\mathrm{GPDH}$, and the triscitrate buffer proved to be superior to Poulik (1957) 

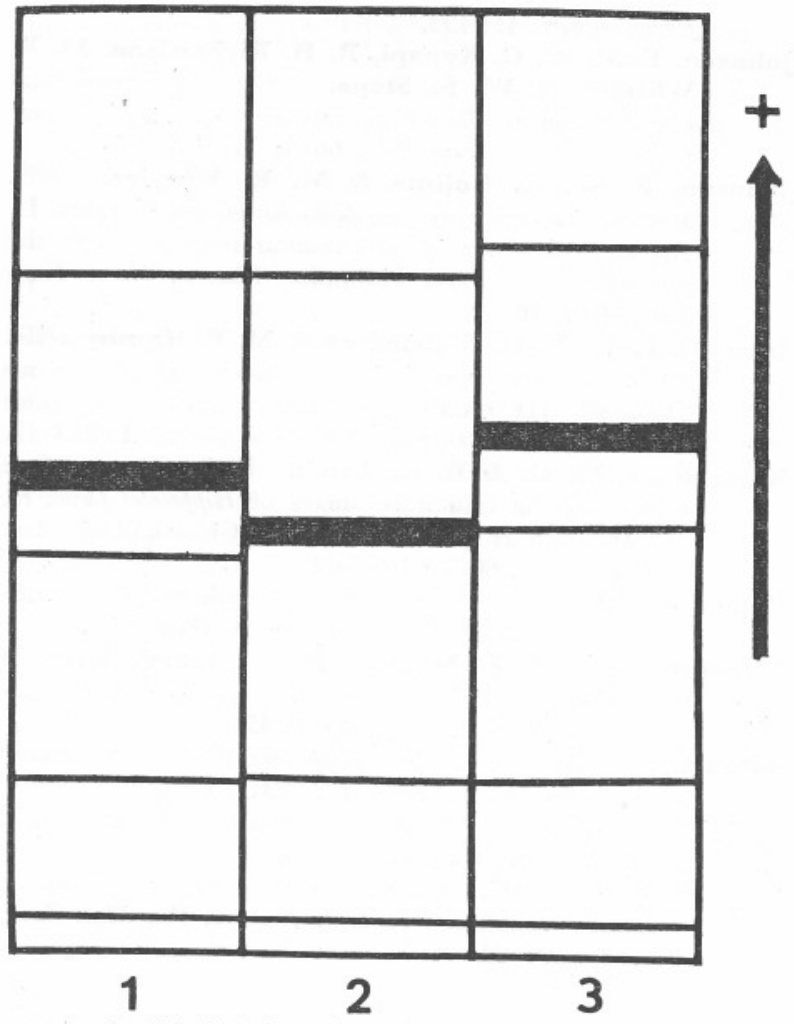

FIG. 2. Typical larval aminopeptidase patterns of $H$. pusio (1), H. bishoppi (2), and $H$. pallipes (3).

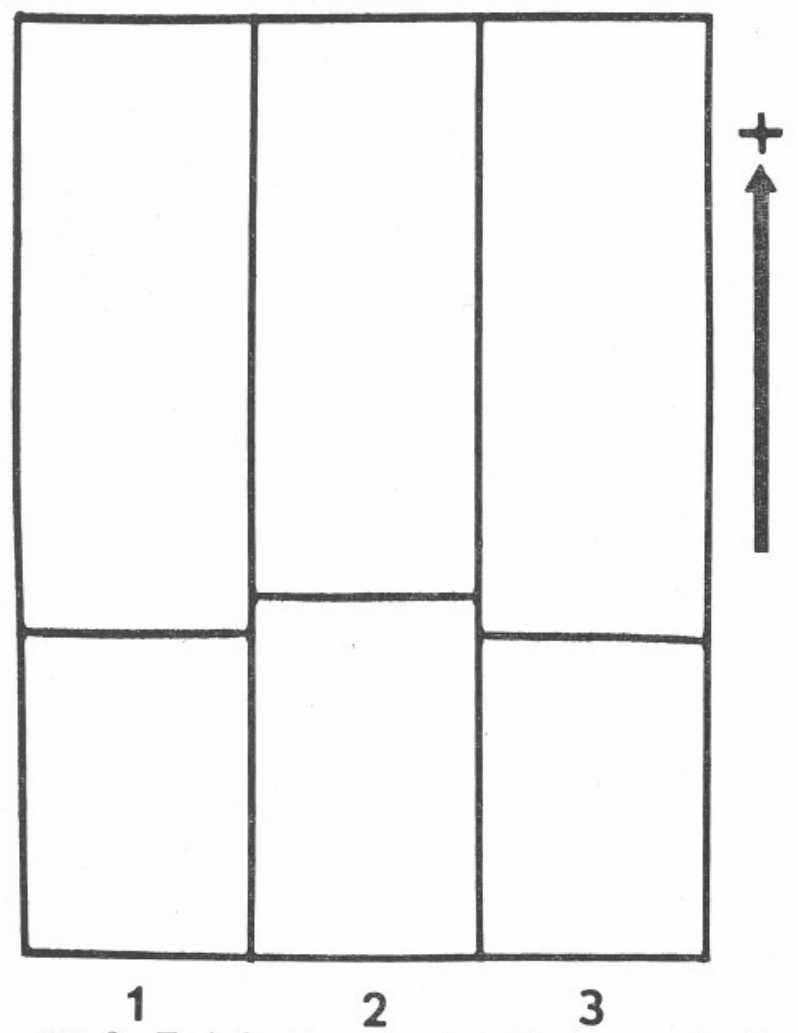

FIG. 3. Typical $\alpha$-glycerophosphate dehydrogenase bands of adults of $H$. pusio (1), H. bishoppi (2), H. pallipes (3).
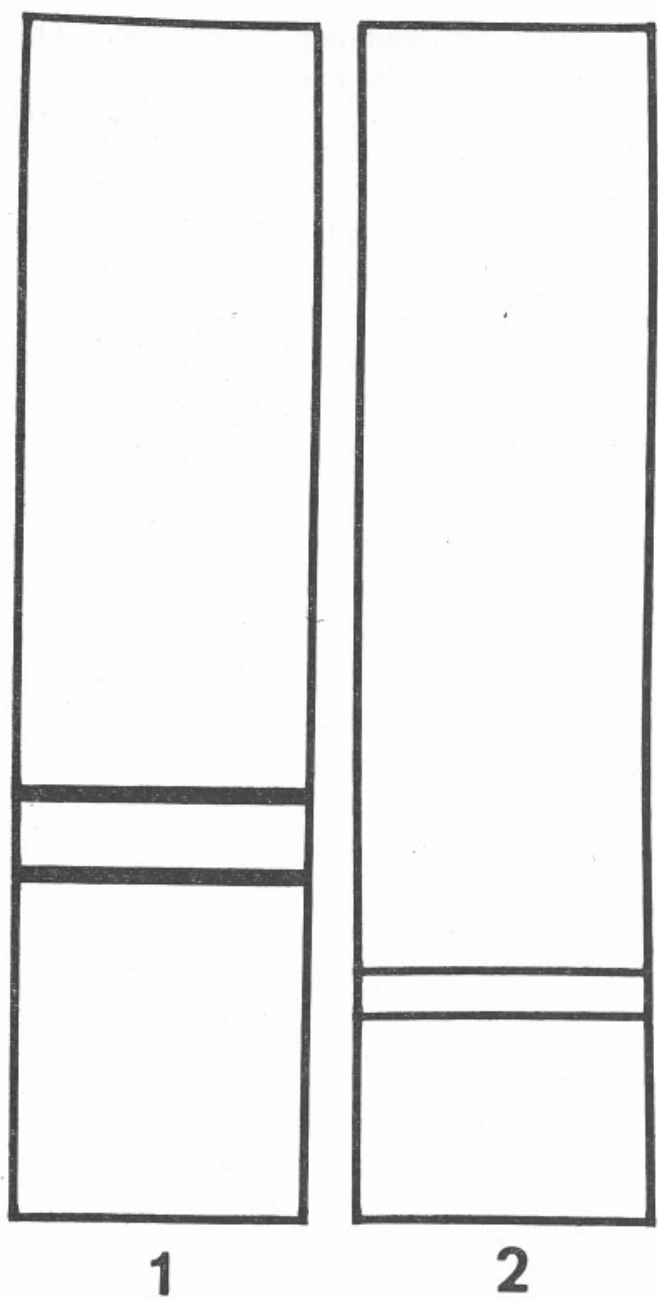

FIG. 4. Diagram of typical NAD-dependent malic dehydrogenase (1) and aldehyde oxidase (2) bands. Identical results were obtained with $H$. pusio, $H$. bishoppi, and $H$. pallipes.

buffer for electrophoretic separation. In H. pusio and $H$. pallipes, the band had the same mobility, but the $\alpha$-GPDH band in $H$. bishoppi had a slightly faster mobility. No intraspecific variation was observed in this system.

For AOX and NAD-dependent $\mathrm{MDH}$ the 3 species had identical patterns (FIG. 4). Gels containing larvae, pupae, and adults could be stained successfully for $\mathrm{MDH}$, but only adults gave acceptable results for AOX. The AOX and $\mathrm{MDH}$ patterns were both double bands of activity, but they migrated differently. The tris-citrate buffer was best for these enzymes.

In all adult assays, no variations due to sex were observed, and only minor activity differences due to age were found.

These results suggest that it is possible to distinguish the 3 species from each other on the basis 
of larval esterases and aminopeptidase alone. The $\alpha$-GPDH apparently can be used to distinguish $H$. bishoppi from the other 2 species. AOX and MDH were, however, uniform in the 3 species. Electrophoretic analysis promises to be useful in analyzing the taxonomically difficult Hippelates. Special care must be taken to sample widely and analyze large numbers of individuals for results to be unequivocal. The present study should be regarded as preliminary until confirmed by more extensive analysis.

Acknowledgments: The assistance of D. Z. Tolar is acknowledged.

\section{REFERENCES CITED}

Axtell, R. C. \& T. D. Edwards. 1970. Seasonal populations of Hippelates gnats (Diptera: Chloropidae) in North Carolina. Ann. Ent. Soc. Amer. 63(4): 1049-53.

Dow, R. P., J. T. Bigham \& C. W. Sabrosky. 1951. Sequel to "Hippelates (eye gnat) investigations in the southeastern states" by John T. Bigham (Diptera: Chloropidae). Proc. Ent. Soc. Wash. 53: 263-71.

Dow, R. P. \& V. D. Hines. 1957. Conjunctivitis in southwestern Georgia. U. S. Publ. Hlth Rep. 72(5): 441-81.

Johnson, F. M. 1966. Rapid single fly homogenization for the investigation of Drosophila isozymes. Drosophila In- formation Serv. 41: 193.

Johnson, F. M., G. G. Kanapi, R. H. Richardson, M. R. Wheeler \& W. S. Stone. 1966. An operational classification of Drosophila esterases for species comparisons. Univ. Texas Publ. 6615: 517-32.

Johnson, F. M., K. Kojima \& M. R. Wheeler. 1969. Isozyme variation in Drosophila island populations. II. An analysis of Drosophila ananassae populations in the Samoan, Fijian, and Philippine Islands. Univ. Texas Publ. 6918: 187-205.

Johnson, F. M., R. H. Richardson \& M. P. Kambysellis. 1968. Isozyme variability in species of the genus Drosophila. III. Qualitative comparison of the esterases of D. aldrichi and D. mulleri. Biochem. Genetics 1: 239-47.

Karandinos, M. G. \& R. C. Axtell. 1967. Temperature effects on the immature stages of Hippelates pusio, $H$. bishoppi, and $H$. pallipes (Diptera: Chloropidae). Ann. Ent. Soc. Amer. 60(5): 1055-62.

Poulik, M. D. 1957. Starch gel electrophoresis in a discontinuous system of buffers. Nature 180: 1477.

Markert, C. L. \& F. Moller. 1959. Multiple forms of esterases: tissue, ontogenetic and species specific patterns. Proc. Natl. Acad. Sci. U. S. 45: 753-63.

Sabrosky, G. W. 1941. The Hippelates flies or eye gnats: preliminary notes. Canad. Ent. 73: 23-27.

1951. Nomenclature of the eye gnats (Hippelates spp.). Amer. J. Trop. Med. 31: 257-58.

Sanders, D. A. 1940. Hippelates flies as vectors of bovine mastitis (preliminary report). J. Amer. Vet. Med. Assoc. 97(763): 306-08. 